\section{Health Equity Does Matter}

The Institute of Population and Public Health (IPPH) of CIHR recently released its 2009-2014 strategic plan titled: "Health Equity Matters". The plan is entirely anchored on a public health imperative to promote health equity and tasks IPPH to provide the tools to support this mission. It identifies four research priorities: 1) improving the understanding of the pathways which promote health equity, 2) determining the impact of complex population health interventions, 3) studying implementation systems for interventions and 4) stimulating theoretical and methodological innovations in knowledge generation, synthesis and transfer. These priorities stem from the recognition of the need to move beyond descriptive studies of health inequities, to the development, implementation and evaluation of real-life solutions. In particular, the field of intervention research is central to the strategic plan. Population health interventions include policies, programs and resources allocation. They are often complex which means that they involve a multitude of players and targets, multiple interventions and delivery mechanisms and are implemented in complex societal systems.

Research in population health interventions is necessarily multidisciplinary, requires substantial resources and time, and will necessitate the development of new theoretical and methodological approaches to intervention design and evaluation. It will also require strong partnerships among practitioners, decision-makers and researchers to ensure that research results are relevant and applicable to the real world. Most interestingly, intervention research will often emerge from "natural experiments", including policies introduced in local jurisdictions, new municipal bylaws, changes in zoning, changes in the physical environment and the numerous laws enacted yearly in the various provinces. Hence, research into implementation systems or delivery mechanisms for population interventions will be critical for the widespread uptake of promising innovations.

Although the absence of global health from the plan is a glaring omission considering that North-South health and economic discrepancies represent one of the most obvious and preventable inequities in the world today, one must salute the judiciousness of the objectives and strategies identified by IPPH as they will provide focused directions for the development of highly relevant research in Canada over the next several years. Despite less than optimal growth prospects in research funding in Canada, I hope that IPPH will be able to continue and expand its successful partnerships with governmental and non-governmental organizations here and abroad to forge an innovative and wide-ranging research agenda that not only is ground-breaking but truly enhances health equity.

Gilles Paradis

Scientific Editor

\section{L'équité en santé est vraiment une nécessité}

L'Institut de la santé publique et des populations (ISPP) des IRSC publiait récemment son plan stratégique 2009-2014 intitulé L'équité en santé : une nécessité. Ce plan s'articule entièrement autour d'un impératif de la santé publique (promouvoir l'équité en santé) et confie à l'ISPP la tâche de fournir les outils qui permettront d'accomplir cette mission. Le plan établit quatre priorités de recherche : 1) mieux comprendre les voies de l'équité en santé, 2) déterminer l'impact d'interventions complexes en santé des populations, 3) examiner les systèmes de mise en œuvre des interventions et 4) stimuler les innovations théoriques et méthodologiques dans la production, la synthèse et le transfert des connaissances. Ces priorités découlent d'un constat : au-delà des études descriptives des inégalités en santé, il faut trouver des solutions pragmatiques, les mettre en œuvre et les évaluer. Le domaine de la recherche d'intervention, en particulier, est au cœur de ce plan stratégique. Les interventions en santé des populations englobent des politiques, des programmes et l'attribution de ressources. Ces interventions sont souvent complexes; autrement dit, elles impliquent une multitude d'acteurs et de cibles, comportent de multiples mesures et modes de prestation et sont mises en œuvre dans des systèmes sociétaux complexes également.

La recherche sur les interventions en santé des populations est nécessairement multidisciplinaire, elle consomme beaucoup de temps et de ressources, et elle nécessitera la mise au point de nouvelles approches théoriques et méthodologiques pour la conception et l'évaluation d'interventions. Elle exigera aussi de solides partenariats entre les praticiens, les décideurs et les chercheurs pour donner des résultats pertinents et pragmatiques. On notera à ce sujet que la recherche d'intervention émerge souvent "d'expériences dans les conditions naturelles » : des politiques lancées au palier local, de nouveaux règlements municipaux, des changements de zonage, des changements dans le milieu physique, ainsi que les nombreuses lois promulguées chaque année dans chaque province. C'est pourquoi la recherche sur les systèmes de mise en œuvre ou sur les modes de prestation des interventions axées sur la population sera d'une importance capitale si l'on veut que les interventions prometteuses se généralisent.

Malgré l'absence évidente de la santé mondiale dans le plan stratégique de l'ISPP (un aspect pourtant incontournable quand on sait que les divergences Nord-Sud au chapitre de la santé et de l'économie sont parmi les inégalités les plus visibles et les plus évitables dans le monde d'aujourd'hui), il faut saluer le choix judicieux des objectifs et des stratégies de l'Institut, qui orienteront le développement de travaux de recherche très pertinents au Canada pendant plusieurs années. Les perspectives de croissance du financement de la recherche au pays sont moroses, mais j'espère que l'ISPP pourra malgré tout poursuivre et élargir ses partenariats fructueux avec les organisations gouvernementales et non gouvernementales canadiennes et étrangères pour forger un programme de recherche vaste et novateur qui ne soit pas seulement original, mais qui améliore vraiment l'équité en santé.

Le rédacteur scientifique, Gilles Paradis 